\title{
Correction to: Semi-equivelar and vertex-transitive maps on the torus
}

\author{
Basudeb Datta ${ }^{1} \cdot$ Dipendu Maity $^{1}$
}

Published online: 15 July 2019

(c) The Managing Editors 2019

\section{Correction to: Beitr Algebra Geom (2017) 58:617-634 https://doi.org/10.1007/s13366-017-0332-z}

If a graph $G$ is embedded on a surface $S$ then the closure of the components of $S \backslash G$ are called faces. If all the faces are 2-disks and intersection of any two intersecting faces is either a vertex or an edge of $G$ then $G$ together with the collection of faces is called a map on $S$. The vertices and edges of $G$ are called the vertices and edges of the map respectively. For a vertex $u$ in a map $M$, the faces containing $u$ form a cycle $C_{u}$ (called the face-cycle at $u$ ) in the dual graph of $M$. A vertex $u$ in a map $M$ is said to be of type $\left[p_{1}^{n_{1}}, p_{2}^{n_{2}}, \ldots, p_{\ell}^{n_{\ell}}\right]$ if the face-cycle $C_{u}$ is of the form $P_{1}-P_{2}-\cdots-P_{\ell}-F_{1,1}$, where $P_{i}=F_{i, 1}-\cdots-F_{i, n_{i}}$ is a path consisting of $p_{i}$-gons $F_{i, 1}, \ldots, F_{i, n_{i}}$ for all $i$. If the types of all the vertices in a map $M$ are $\left[p_{1}^{n_{1}}, p_{2}^{n_{2}}, \ldots, p_{\ell}^{n_{\ell}}\right]$ then we say the map $M$ is semi-equivelar of type $\left[p_{1}^{n_{1}}, p_{2}^{n_{2}}, \ldots, p_{\ell}^{n_{\ell}}\right]$.

Part (iii) of Lemma 2.2 in the orginal article is not true in general. For example, by Lemma 2.2 (iii), there can not exist a map of type $\left[p^{2}, q^{1}, p^{1}, r^{1}\right]$, where $p, q, r$ are distinct and $p$ is odd. But, there exists a map of type $\left[3^{2}, 4^{1}, 3^{1}, 6^{1}\right.$ ] [see (Karabáš and Nedela 2012, Example $A_{2.93}$, Page 581)]. In the proof of Lemma 2.2 (iii) in the orginal article, it was implicitly assumed that $p_{j} \neq p_{i}$ for all $j \neq i$. Here we present a replacement of part (iii) of (Lemma 2.2 in the orginal article). For the sake of completeness, we are presenting the statements of the other two parts also.

Lemma 2.2'. If $\left[p_{1}^{n_{1}}, \ldots, p_{k}^{n_{k}}\right]$ satisfies any of the following three properties then $\left[p_{1}^{n_{1}}\right.$, $\left.\ldots, p_{k}^{n_{k}}\right]$ can not be the type of any semi-equivelar map on a surface.

The original article can be found online at https://doi.org/10.1007/s13366-017-0332-z.

Basudeb Datta

dattab@iisc.ac.in

Dipendu Maity

dipendumaity@iisc.ac.in

1 Department of Mathematics, Indian Institute of Science, Bangalore 560 012, India 
(i) There exists $i$ such that $n_{i}=2, p_{i}$ is odd and $p_{j} \neq p_{i}$ for all $j \neq i$.

(ii) There exists $i$ such that $n_{i}=1, p_{i}$ is odd, $p_{j} \neq p_{i}$ for all $j \neq i$ and $p_{i-1} \neq p_{i+1}$. (Here, addition in the subscripts are modulo $k$.)

(iii) $\left[p_{1}^{n_{1}}, \ldots, p_{k}^{n_{k}}\right]$ is of the form $\left[p^{1}, q^{m}, p^{1}, r^{n}\right]$, where $p, q$, $r$ are distinct and $p$ is odd.

Proof Since Parts (i) and (ii) are the same as in (Lemma 2.2 in the orginal article) and true, we are presenting the proof of part (iii) only.

Assume that there exists a semi-equivelar map $Z$ of type $\left[p^{1}, q^{m}, p^{1}, r^{n}\right]$, where $p, q, r$ are distinct and $p$ odd. Let $P$ and $Q$ be two adjacent faces at a vertex $u_{1}$, where $P$ is a $p$-gon and $Q$ is a $r$-gon. Assume that $P=u_{1}-u_{2}-u_{3}-\cdots-u_{p}-u_{1}$ and $Q=u_{1}-v_{2}-v_{3}-\cdots-v_{r-1}-u_{p}-u_{1}$. Let the other face containing $u_{j} u_{j+1}$ be $P_{j}$ for $1 \leq j \leq p$. (Additions in the subscripts are modulo $p$.) Since $p, q, r$ are distinct, considering the face-cycle of $u_{1}$, it follows that $P_{1}$ is a $q$-gon. Considering the face-cycle of $u_{2}$, by the similar argument (interchanging $r$ and $q$ ), it follows that $P_{2}$ is a $r$-gon. Continuing this way, we get $P_{1}, P_{3}, \ldots$ are $q$-gons and $P_{2}, P_{4}, \ldots$ are $r$-gons. Since $p$ is odd, it follows that $P_{p}$ is a $q$-gon. This is a contradiction since $P_{p}=Q$ is a $r$-gon and $r \neq q$. This completes the proof.

In the orginal article, we used Lemma 2.2 (iii) to prove non-existence of a map of type $\left[3^{1}, 4^{1}, 3^{1}, 12^{1}\right]$ (see the proof of Theorem 1.4 in the original article). This now follows from part (iii) of Lemma 2.2' above. Therefore, all the results (except part (iii) of Lemma 2.2) in the orginal article are correct.

Acknowledgements The authors thank Agneedh Basu for pointing out this error. The authors also thank the anonymous referee for pointing out some corrections.

\section{Reference}

Karabáš, J., Nedela, R.: Archimedean maps of higher genera. Math. Comput. 81(277), 569-583 (2012)

Publisher's Note Springer Nature remains neutral with regard to jurisdictional claims in published maps and institutional affiliations. 